\title{
Aerostructural Optimization of Long Span Bridges: Current Advances and Challenges
}

\author{
Santiago Hernandez, F.ASCE${ }^{1}$; Felix Nieto, M.ASCE ${ }^{2}$; Miguel Cid Montoya, Ph.D. ${ }^{3}$; \\ and José A. Jurado ${ }^{4}$ \\ ${ }^{1}$ Prof., School of Civil Engineering, Univ. of Coruña, Campus Elviña, Spain. E-mail: \\ hernandez@udc.es \\ ${ }^{2}$ Ass. Prof., School of Civil Engineering, Univ. of Coruña, Campus Elviña, Spain. E-mail: \\ fnieto@udc.es \\ ${ }^{3}$ School of Civil Engineering, Univ. of Coruña, Campus Elviña, Spain; Fulbright Visiting \\ Scholar, NatHaz Modeling Laboratory, Univ. of Notre Dame, IN, USA. E-mail: \\ miguel.cid.montoya@udc.es \\ ${ }^{4}$ Ass. Prof., School of Civil Engineering, Univ. of Coruña, Campus Elviña, Spain. E-mail: \\ jjuradoo@udc.es
}

\begin{abstract}
This paper describes the evolution of deck shape of long span bridges since the Tacoma Narrows collapse trying to avoid undesirable aerodynamic behavior under wind flow and the trend in the last decades to increase the length of the main span of suspension and cable stayed bridges. The necessity to use advanced technologies to help the engineer to obtain the best possible design is highlighted and the advantages of applying optimization methodologies is encouraged. It is explained that this approach requires to use only numerical tools and hence to eliminate experimental studies, as wind tunnel tests using reduced models of full bridge of a segment of the deck, and their substitution by computational fluid dynamics (CFD) simulations. After doing so, the current capabilities of this approach are presented and, finally, the problems that need to be solved to have a fully operational methodology able to be implemented in real structures are outlined.
\end{abstract}

\section{INTRODUCTION}

The well known collapse of the Tacoma Narrows Bridge in 1940 under a mild storm with wind speed of about $18 \mathrm{~m} / \mathrm{s}$ created a severe problem for bridge engineers. This suspension bridge had been designed by a prestigious engineer using the more recent theory of structural analysis, the entitled deflexion theory, and selecting a deck shape that, at that time, was supposed to be very efficient. As the failure put in evidence, civil engineers did not have at that time proper knowledge of the loads induced by wind flow in suspension bridges and the bridge deck shape, composed by two longitudinal beams with some traverse connections, behaved worse than the stiff trusses previously used for the girder in this class of structures.

This situation led to the so called Tacoma effect, that induced bridge engineers to design suspension bridges more cautiously by returning to support the deck with trusses, In the words of a researcher "the biggest casualty (of Tacoma failure) was a generation of engineering practice.." (Scott 2001). The event also generated a strong research impulse aimed to find out better theories for understanding wind induced phenomena in general, and the effects of flutter in bridges in particular, and to create new shapes for bridge decks, able to deal more efficiently with aerodynamic loads.

The new bridge deck adopted was a very shallow closed box, that was soon coined as streamlined box and was first used in the suspension bridge over the estuary of the Severn bridge 
in UK in 1960 (Roberts 1968, Brown 1975a). The great success of this bridge deck originated that this geometry has been used in almost all relevant suspension bridges built since then, as in the cases of the Humber (UK) and Bosphorus (Turkey) bridges (Brown 1975b), the Great Belt Bridge (Denmark) or the most recent Osman Gazi and Yavuz Sultan Selim (Turkey) bridges, that have been designed with aerodynamic decks. The only exception is the Akashi Bridge that has a truss girder due to the high stiffness required to undergo both strong earthquakes and aerodynamic loads.

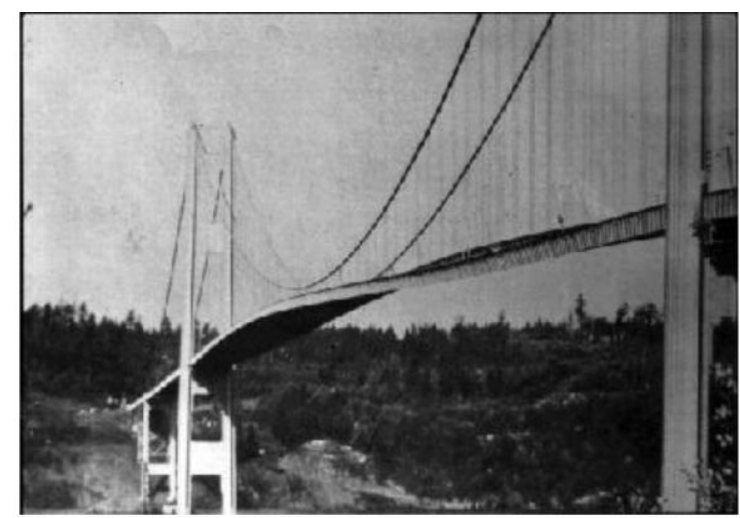

a) First Tacoma Narrows Bridge (www.txstate.edu)

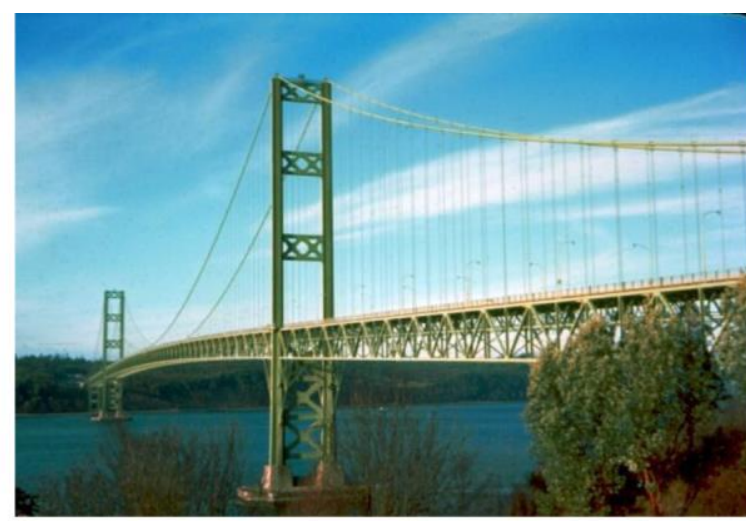

b) Second Tacoma Narrows Bridge (commons. wikipedia.org)

Figure 1: Images of the Tacoma Narrows bridges

The long span cable stayed bridges built in the last two decades have made use also of the aerodynamic decks and the Normandy (France), Tatara (Japan) and Incheon (South Korea) bridges are examples with central span lengths in the range of 800-900 $\mathrm{m}$ while the Sutong (China) and Russky (Russia) bridges with main span longer than $1 \mathrm{~km}$ are the most prominent examples.

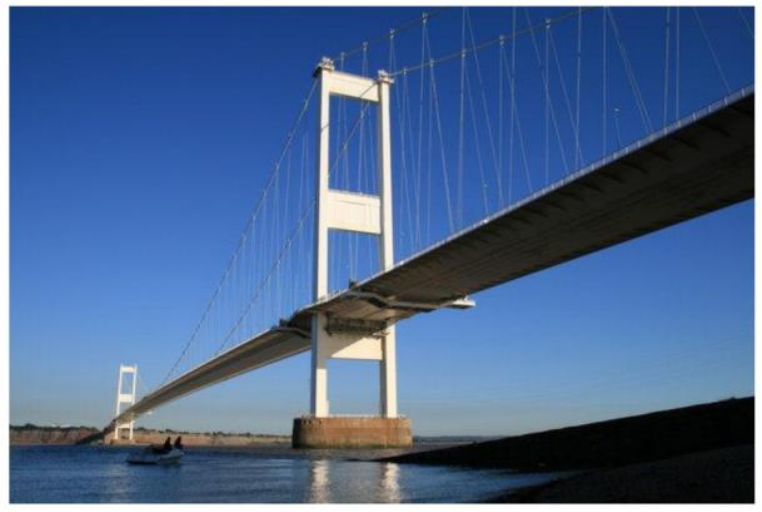

a) Picture of the Severn bridge (www.geograph.org.uk)

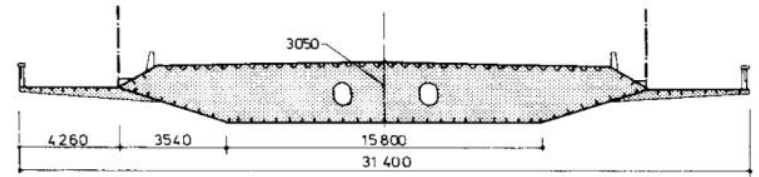

b) Detail of the streamlined deck shape (Cable supported bridges. N. J. Gimsing)

Figure 2. Severn Bridge

The trend of increasing length in central span of both suspension and cable stayed bridges has made that the single box deck is reaching its upper threshold of efficiency and thus new geometries composed by two separate boxes have been created for suspension bridges as the 
Sihoumen (China) and for cable stayed bridges as the Edong and Stonecutters bridges in that country, to enhance the aeroelastic performance of the bridge.

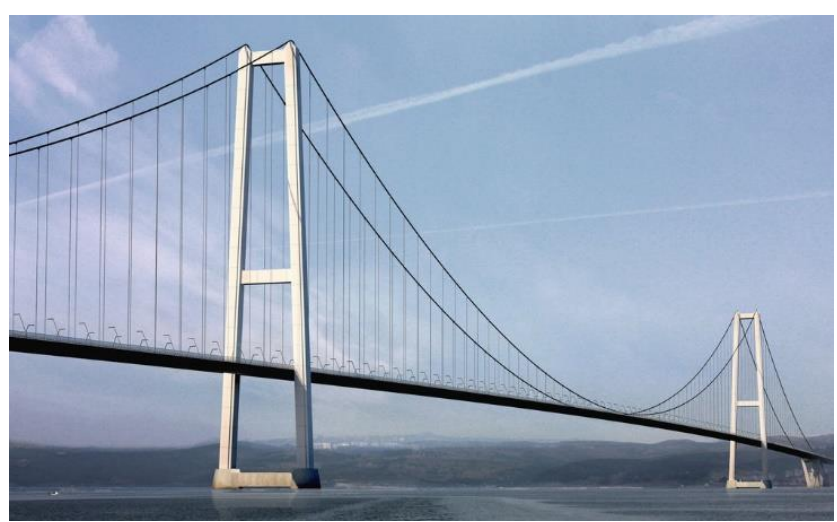

Figure 3. Osman Gazi Bridge (www.dw.dk)

The research on bridge aeroelasticity, aimed to understand the interaction between bridge deformation and wind flow, made an outstanding advance with the Scanlan's formulation (Scanlan and Tomko 1971) that established a relationship between aeroelastic wind forces generated for laminar wind flow, and deck displacements and velocities through a set of eighteen functions called flutter derivatives, that were function of wind speed and bridge vibration frequency.

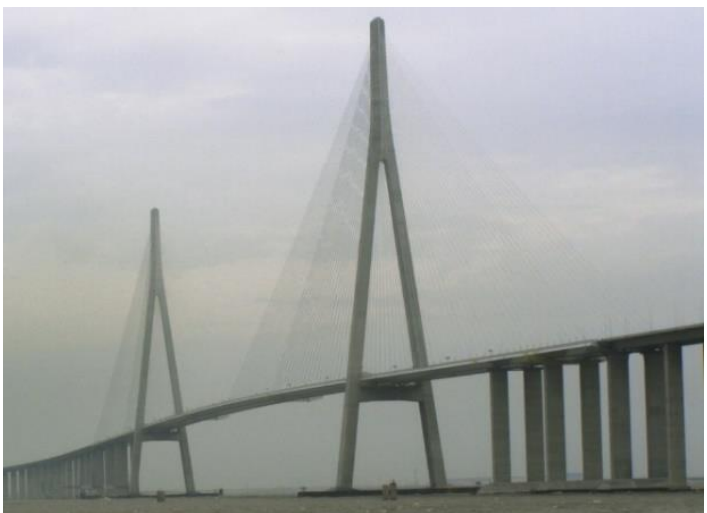

Figure 4. Sutong Bridge (commons.wikipedia.org)

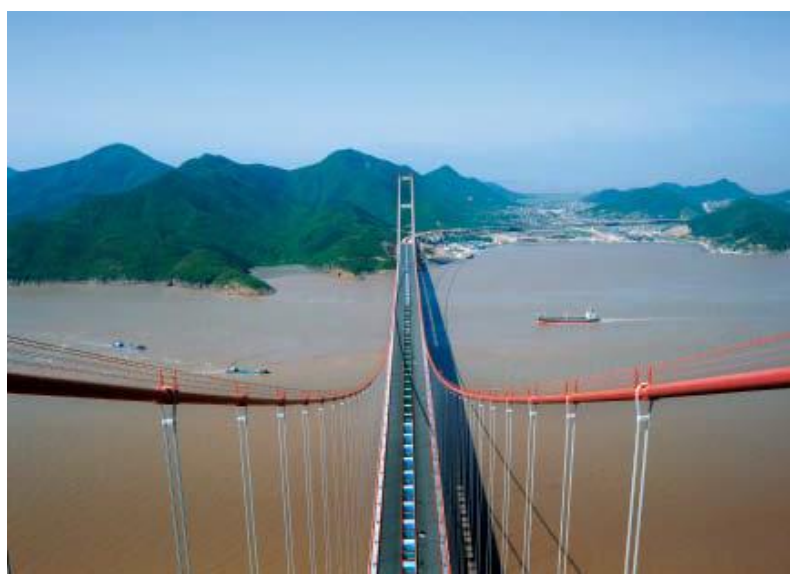

Figure 5. Sihoumen Bridge (china.bridge.org.cn) 
This technique allowed to substitute wind tunnel testing of reduced models of the full bridge by a more inexpensive test of a reduced model of a deck segment that provided the flutter derivatives. Afterwards, in a second part of the study, a computer analysis of the finite element model of the full bridge subject to the aeroelastic forces provided the flutter speed, the speed of wind flow that initiates the flutter phenomenon.

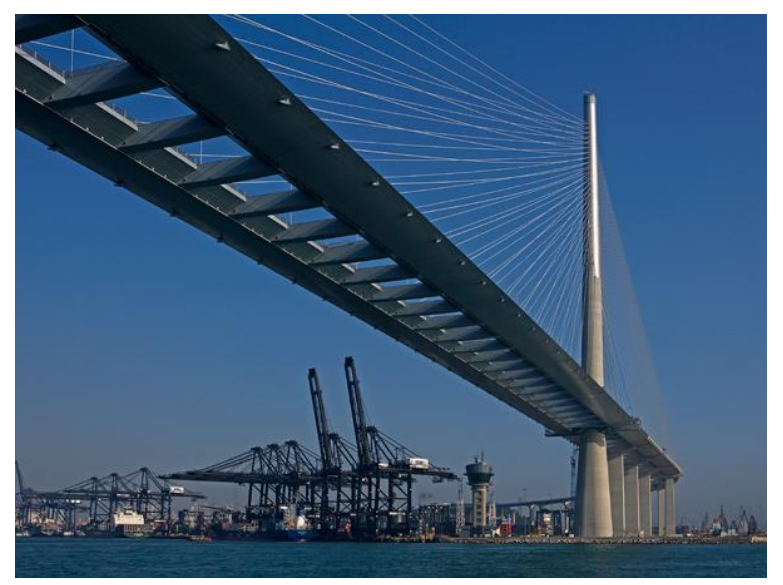

Figure 6. Stonecutters Bridge (word-architects.com)

This approach opened the door for computer based formulations of other aerodynamic phenomena as torsional divergence, to include the effects of turbulent wind producing buffeting and to consider also vortex shedding phenomenon.

Nevertheless, in spite of the continuous increment of complexity of suspension and cable stayed bridges the design was been made by the conventional method of trial and error techniques, adjusting the design by using rules mainly based in engineering judgements and intuition, all of this in the common situation of limited time and available resources.

This situation could be improved by using the capabilities of algorithms of numerical optimization that can identify the solution that accomplishes more perfectly the set of conditions stated by the requirements plan of the project. The methodologies of optimum design have been used for many years in aircraft and car engineering but only recently have been incorporated in civil engineering. This is clear that the design of such challenging structures as long and ultralong cable stayed or suspension bridges subject to wind induced loads could benefit by making use of such techniques and the approach to implement such methodology, the findings obtained so far and the remaining issues that need to be solved to obtain a very reliable formulation will be described in the following sections.

\section{TECHNOLOGIES INVOLVED IN NUMERICAL AEROSTRUCTURAL OPTIMIZATION OF LONG SPAN BRIDGES}

The objective of the aerostructural optimization of long span bridges in to find out the shape of the deck and the dimensions of the cable system that minimizes the weight of the bridge while accomplishing the requirements associated to dead or service loads like, for instance the stresses in the material or the displacements of the structure and also the conditions related to wind induced phenomena as instabilities like flutter or torsional divergence or to the values of the displacements produced by turbulent flow in the cases of buffeting or vortex shedding.

Having explained that, the disciplines involved in this approach are 
1) Numerical formulation of wind induced phenomenon forces: The forces produced by the wind flow will need to be expressed in a way that can be implemented in computer codes.

2) Application of Computational Fluid Mechanics (CFD) simulations of several geometries of bridge decks under wind flow that provide the information that was usually obtained in wind tunnel test.

3) Generation of surrogate models that contain the results of the CFD simulations and allow inferring appropriately the dynamic properties of other deck geometries.

4) Generation of finite element models of the full bridge to be used in calculations of the structure under static and aerodynamic loads.

5) Assembly of these pieces of information in the manner required in the formulation of methods of design optimization and application of numerical optimization algorithms that will provide the best solution to the problem.

\section{NUMERICAL FORMULATION OF WIND INDUCED FORCES}

Forces related to torsional divergence, flutter, buffeting or vortex shedding need to be formulated by expressions that can be applied easily in finite element models of the bridge.

Torsional divergence: It is well known that consist in an instability produced by an ever increasing twisting angle produced by moment created by the wind flow. This behavior has been studied in aircraft engineering considering the twisting moment and the rotation produced by wind, but in bridges the structure needs to be studied not only under the twisting moment $M$, but also under the horizontal $D$ (drag) and vertical $L$ (lift) load components. Because of that, in bridge engineering this phenomenon receives also the name of aeroelastic instability. Figure 7 shows the forces and the rotation of a bridge deck.

The expression that relates the vector of twisting angle of the nodes of the deck $\boldsymbol{\varphi}_{x}$ to the flexibility matrix $\mathbf{F}$ and forces vector $\mathbf{f}$ is

$$
\boldsymbol{\varphi}_{x}=\mathbf{F f}\left(\boldsymbol{\varphi}_{x}\right)
$$

As forces $\mathbf{f}$ are function of the wind speed $U$ and vector angles $\boldsymbol{\varphi}_{x}$, expression (1) leads to an eingenvalue problem. The last eigenvalue provides the wind speed that generate the aerostatic instability.

Flutter: Scanlan's formulation is the common technique to formulate the self-excited forces produced when the bridge vibrates under wind of constant speed $U$ that can lead to flutter instability. Forces are dependent of wind speed $U$, vibration frequency $\omega$, displacements, $v, w$ and $\varphi_{x}$ and velocities, $\dot{v}, \dot{w}, \dot{\varphi}_{x}$, of the deck. Per unit length the expression of the forces is.

$$
\left(\begin{array}{l}
D \\
L \\
M
\end{array}\right)=\frac{1}{2} \rho U^{2}\left(\begin{array}{ccc}
K^{2} P_{4}^{*} & -K^{2} P_{6}^{*} & -B K^{2} P_{3}^{*} \\
-K^{2} H_{6}^{*} & K^{2} H_{4}^{*} & B K^{2} H_{3}^{*} \\
-B K^{2} A_{6}^{*} & B K^{2} A_{4}^{*} & B^{2} K^{2} A_{3}^{*}
\end{array}\right)\left(\begin{array}{c}
v \\
w \\
\varphi_{x}
\end{array}\right)+\frac{1}{2} \rho U^{2}\left(\begin{array}{ccc}
B K P_{1}^{*} & -B K P_{5}^{*} & -B^{2} K P_{2}^{*} \\
-B K H_{5}^{*} & B K H_{1}^{*} & B^{2} K H_{2}^{*} \\
-B^{2} K A_{5}^{*} & B^{2} K A_{1}^{*} & B^{3} K A_{2}^{*}
\end{array}\right)\left(\begin{array}{c}
\dot{v} \\
\dot{w} \\
\dot{\varphi}_{x}
\end{array}\right)
$$

Functions $A_{i}, H_{i}, P_{i},(i=1, \ldots, 6)$ are the flutter derivatives and depend also of wind speed and vibration frequency. Assembling the expression at bridge level, it turns out

$$
\mathbf{M u ̈}+\mathbf{C u}+\mathbf{K u}=\mathbf{f}_{a}=\mathbf{K}_{a} \mathbf{u}+\mathbf{C}_{a} \dot{\mathbf{u}}
$$

Again, this equation leads to a nonlinear eigenvalue problem that provides the wind speed that corresponds to the vibration frequency that cancels out the value of the damping.

Buffeting: Forces produced by the turbulent component of along wind speed $u$ and vertical speed $w$, are expressed as indicated in (4) where $\chi_{D u}^{*}, \chi_{D w}^{*}, \chi_{L u}^{*}, \chi_{L w}^{*}, \chi_{M u}^{*}$ and $\chi_{M w}^{*}$ are the 
admittance functions.

$$
\left\{\begin{array}{c}
D_{b u f f} \\
L_{b u f f} \\
M_{\text {buff }}
\end{array}\right\}=\frac{1}{2} \rho U^{2} B\left[\begin{array}{cc}
\chi_{D u}^{*} & \chi_{D w}^{*} \\
\chi_{L u}^{*} & \chi_{L w}^{*} \\
B \chi_{M u}^{*} & B \chi_{M w}^{*}
\end{array}\right]\left\{\begin{array}{l}
\frac{u}{U} \\
\frac{w}{U}
\end{array}\right\}
$$

The dynamic equilibrium of the structure in formulated considering the laminar and turbulent wind flow. Thus, at bridge level the equation is

$$
\mathbf{M u ̈}+\mathbf{C} \dot{\mathbf{u}}+\mathbf{K u}=\mathbf{K}_{a} \mathbf{u}+\mathbf{C}_{a} \dot{\mathbf{u}}+\mathbf{f}_{\text {buff }}
$$

The dynamic analysis is carried out in the frequency domain applying the Fourier transform to equation (5) and using power spectral density (PSD) expressions to characterize the wind flow. The PSD of the responses of the bridge provides the root mean square (RMS) of displacements, velocities and accelerations of the nodes of the structural model that are the responses of interest for the design of the bridge.

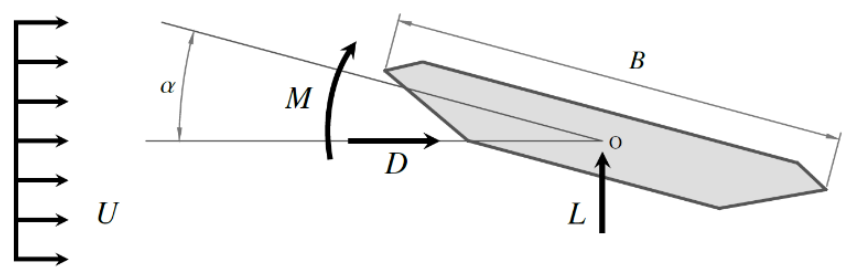

Figure 7. Sign convention of forces and rotation for the studies of aeroelastic instability

Vortex shedding: Any elastic bluff body immersed in a fluid produces vortex of alternating gyration axis that produce an across wind force of also, alternating sense and therefore an across wind vibration of the body. The frequency of these vortexes are linear dependent of wind speed and the range of displacements produced are small. Nevertheless if, for a given wind speed, the frequency of the vibration reaches a natural frequency of the body the amplitude of the displacements increases substantially and the frequency of the vortex does not change although wind speed increases. This phenomenon is denominated lock-in and it is undesirable because it shorten the duration of service life due to fatigue considerations. Therefore, the process of bridge design needs to prevent this phenomenon.

The expression of the dynamic equilibrium of the structure in the vortex shedding studies in written in expression (6) (Vickery and Basu 1983). It can be understood that in the proximity of the lock-in the damping matrix $\mathbf{C}$ of the structure is reduced by the component $\mathbf{C}_{V I V}$ and $\mathbf{f}_{V I V}(\mathrm{t})$ are the forces produced by the vortex.

$$
\mathbf{M} \ddot{\mathbf{u}}+\left(\mathbf{C}-\mathbf{C}_{V I V}\right) \dot{\mathbf{u}}+\mathbf{K u}=\mathbf{f}_{V I V}(\mathbf{t})
$$

As in the case of the buffeting, the dynamic analysis is carried out in the frequency domain applying the Fourier transform to equation (6) and using power spectral density (PSD) expressions to characterize the wind flow. The PSD of the responses of the bridge provides the root mean square (RMS) of displacements, velocities and accelerations of the nodes of the structural model that are the responses of interest for the design of the bridge.

\section{COMPUTATIONAL FLUID MECHANICS ANALYSIS OF BRIDGE DECKS}

As mentioned before the identification of the aeroelastic properties of bridge decks was done in the past by means of wind tunnel test of reduced models of deck segments that provide the 
expressions of the aerodynamic coefficients of the flutter derivatives. Nevertheless, the advances in computational formulations of fluid dynamics problems have made possible to use numerical techniques to obtain these information. Computational Fluid Dynamics simulations consist on recreating the behavior of the wind flow passing through an elastic body immersed in it and the existing interaction. Currently there are two different formulations.

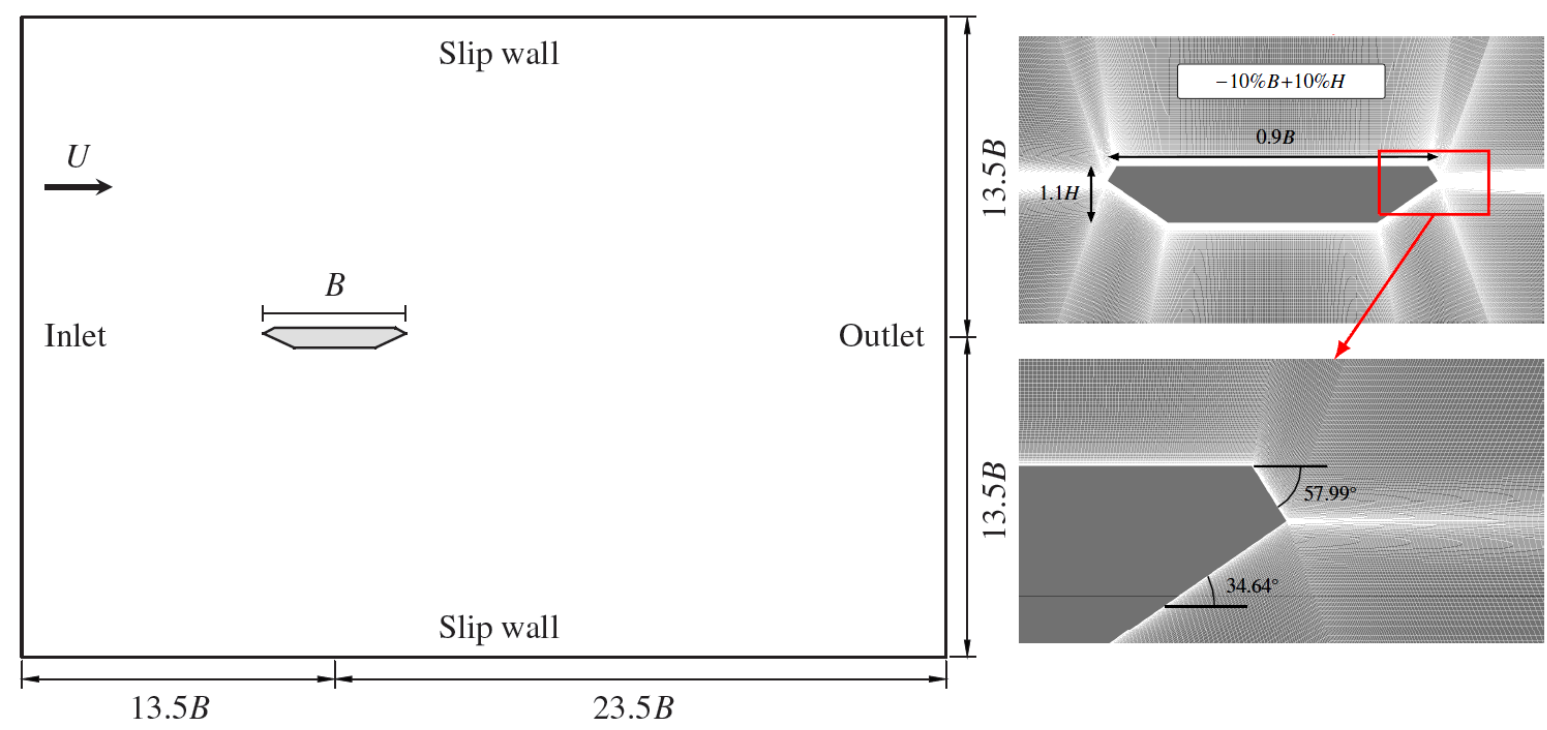

Figure 8. Domain of study and detail of the body and the mesh of finite volumes.

Domain discretization by finite volumes: This methodology deals with the Navier Stokes (NS) equations and discretizes the domain under study creating a number of finite volumes defined by vertex and lines. The set of differential equations of NS is transformed in a system of linear equations whose unknowns are the characteristics of the flow at the domain vertex.

Several approaches exist (RANS, URANS, LES and DES), and are useful in aerodynamic studies of airfoils or wind mill blades, but their efficiency in bridge aeroelasticity needs to be improved, in other words, while the calculation of the aerodynamic coefficients is made very accurately, the situation with respect the flutter derivatives is less productive, as there are discrepancies amongst the numerical results provided by the different CFD turbulence models, and usually they are also different of the data obtained in the wind tunnel test of reduced models of deck segments (Bruno et al., 2014).

Lattice-Boltzmann methods (LBM): In this technique, a number of fictitious particles defined in the domain move and collide amongst them accomplishing the Boltzmann transport equation. Again, in this approach the differential equations become a set of linear equations. This methods is been used nowadays in aircraft engineering but it is almost unused in bridge aeroelasticity.

A scheme of the domain used in a CFD simulation and some details of the mesh of finite volumes is presented in Figure 8.

In both methods, a verification study aimed to observe the accuracy of the results is necessary. That means to generate CFD models with different level of complexity and refinement, for instance dimensions of the domain, Reynolds number considered, mesh size, number of nodes, number of particles, and so on.

Two main classes of information are needed as outcome of the CFD simulations of the bridge deck for their use in the aeroelastic studies of the full bridge 
1) Aerodynamic coefficients $C_{D}, C_{L}, C_{M}$ and their derivatives at $0^{\circ}$ angle of attack of wind flow

2) Flutter derivatives $H_{i}, A_{i}, P_{i}(i=1, . ., 6)$

The current capabilities of CFD methods allow evaluating very efficiently the aerodynamic coefficients for a wide interval of angle of attack of the flow (Álvarez et al. 2018) and thus, their derivatives with horizontal wind are also accurate. On the other hand, the evaluation of the flutter derivatives is more complicated and computationally expensive and the level of precision poorer (Nieto et al. 2015). Because of that, an alternative approach is to use the so-called quasi steady theory (QST) that provides a subset of the flutter derivatives by using the aerodynamic coefficients and their derivatives. The relationship appears in expression (7)

$$
\begin{aligned}
& H_{1}^{*}=-\frac{C_{L}^{\prime}+C_{D}}{K}, \quad H_{2}^{*}=\frac{C_{L}^{\prime}+C_{D}}{K} \mu_{H}, H_{3}^{*}=-\frac{C_{L}^{\prime}}{K^{2}}, H_{5}^{*}=-\frac{2 C_{L}}{K} \\
& A_{1}^{*}=\frac{C_{M}^{\prime}}{K}, A_{2}^{*}=\frac{C_{M}^{\prime}}{K} \mu_{A}, A_{3}^{*}=\frac{C_{M}^{\prime}}{K^{2}}, \quad A_{5}^{*}=-\frac{2 C_{M}}{K} \\
& P_{1}^{*}=-\frac{2 C_{D}}{K}, \quad P_{2}^{*}=\frac{C_{D}^{\prime}-C_{L}}{K} \mu_{P}, P_{3}^{*}=\frac{C_{D}^{\prime}}{K^{2}}, P_{5}^{*}=-\frac{C_{D}^{\prime}-C_{L}}{K}
\end{aligned}
$$

Where $\mu_{H}$ and $\mu_{A}$ can be obtained by the following expressions (Larose and Livesey, 1997)

$$
\mu_{H} \approx \frac{A_{1}^{*}}{H_{1}^{*}} \text { and } \mu_{A} \approx \frac{A_{3}^{*}}{H_{3}^{*}}
$$

And $\mu_{P}$ is assumed to take the same value as $\mu_{H}$.
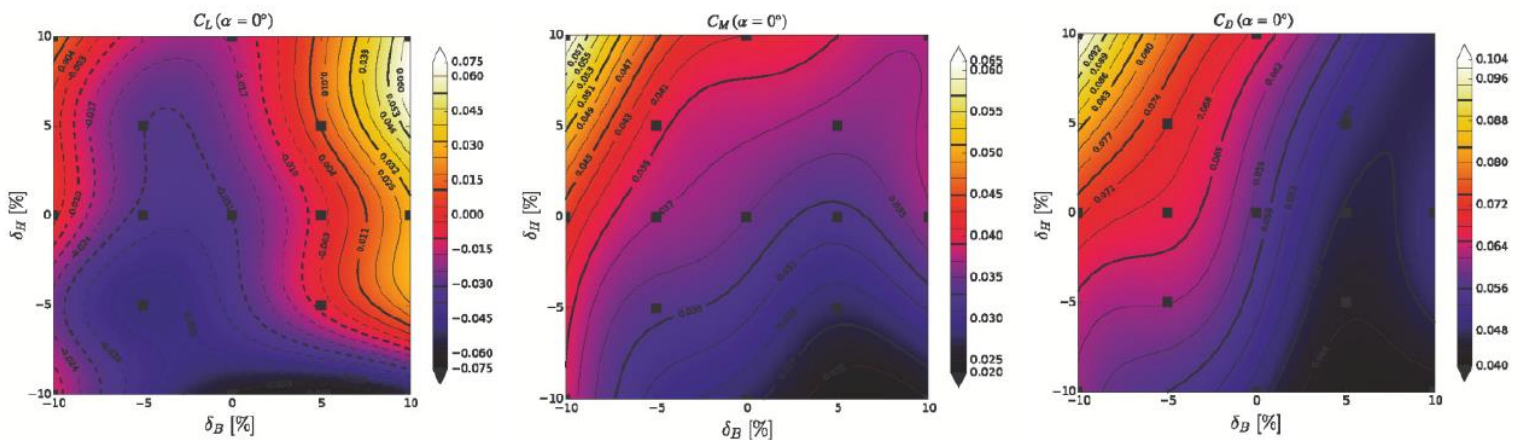

Figure 9. Examples of surrogate model (Cid Montoya et al. 2018a).

\section{SURROGATE MODELS}

The essence of the optimization methods is the fact that the values of the design variables change along the process and consequently the structural and aeroelastic analysis carried out at each iteration need to be done with the aerodynamic properties of the deck, associated to these new values. As they are not known in advance it is necessary to generate a method that provides the aerodynamic properties of the deck for any geometry inside the allowed range of variation. This is done by generating a number of CFD simulations with different values of the design variables that cover appropriately enough the design domain.

This can be done in a deterministic manner, selecting the geometries of the deck to be chosen for the simulations, or making use of design of experiments (DoE) techniques that guarantee the 
simulations cover the complete domain of the design variables in an efficient mode. The results of the CFD analysis allow to create a surrogate model (Forrester et al., 2008, Cid Montoya et al. 2018a, Nieto et al. 2019) that is, in fact, a function that provides the necessary data of the aerodynamic properties for any value of the design variables during the optimization process. Some graphical examples of representations of surrogate models appear in Figure 9.

\section{FINITE ELEMENTS MODELS OF BRIDGES}

The complete design of a bridge requires a number of structural models whose properties differ depending of the purpose of each specific step or task. In the case of aeroelastic studies models like those presented in Figure 10 are used.
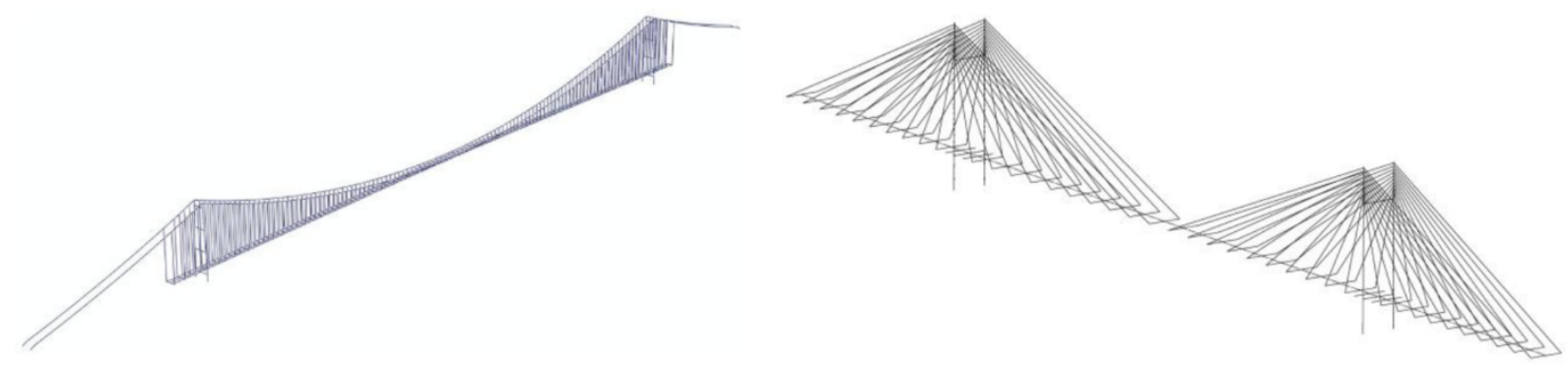

Figure 10. Finite element models of long span cable supported bridges

It can be observed that in both cases the deck is defined by beam elements that have the aerodynamic properties of the deck. These models also serve for eigenvalues calculations and for the static analysis for dead or service loads because the FEM provides the displacements of the nodes and the stress analysis in the towers, cables and deck can be carried out from data of area and inertia moduli of these structural components.

\section{FORMULATION OF AEROSTRUCTURAL OPTIMIZATION OF BRIDGES}

The disciplines presented in the previous paragraph are the pieces necessary to formulate the aerostructural optimization of bridges, with the aim of identify the deck shape and the size of the elements of the cable system that requires the minimum amount of material for the complete bridge, subject to forces coming from self-weight, service loads and the aeroelastic phenomena produced by laminar and turbulent wind flow. Therefore, the optimization problem is formulated as follows

1) Design variables:

i) Deck shape variables $H, B$

ii) Vector of thicknesses of deck plates $t$

iii) Vector of areas of cables $\boldsymbol{A}$ and of prestressing forces $\boldsymbol{N}$

2) Structural constraints:

i) Stresses in deck and cables.

ii) Vertical displacements of deck

iii) Horizontal displacements of the top node of the towers

3) Aeroelastic constraints:

i) Flutter speed

ii) Speed of torsional divergence (aerostatic instability)

iii) RMS of deck accelerations or displacements produced by turbulent flow 
iv) RMS of deck accelerations or displacements near of the lock-in wind speed

4) Objective function: Total amount of material for deck and cable system

This approach has several advantages because it manages simultaneously considerations of very two separate fields as structural and aeroelastic ones. It may be remembered that in the conventional design process only structural limitations are studied and only when the design of the bridge is mature enough some test are carried out in wind tunnels to check the appropriateness of the prototype. If any undesired behavior appears, the necessary modifications need to be made and this loop can be cumbersome and costly.

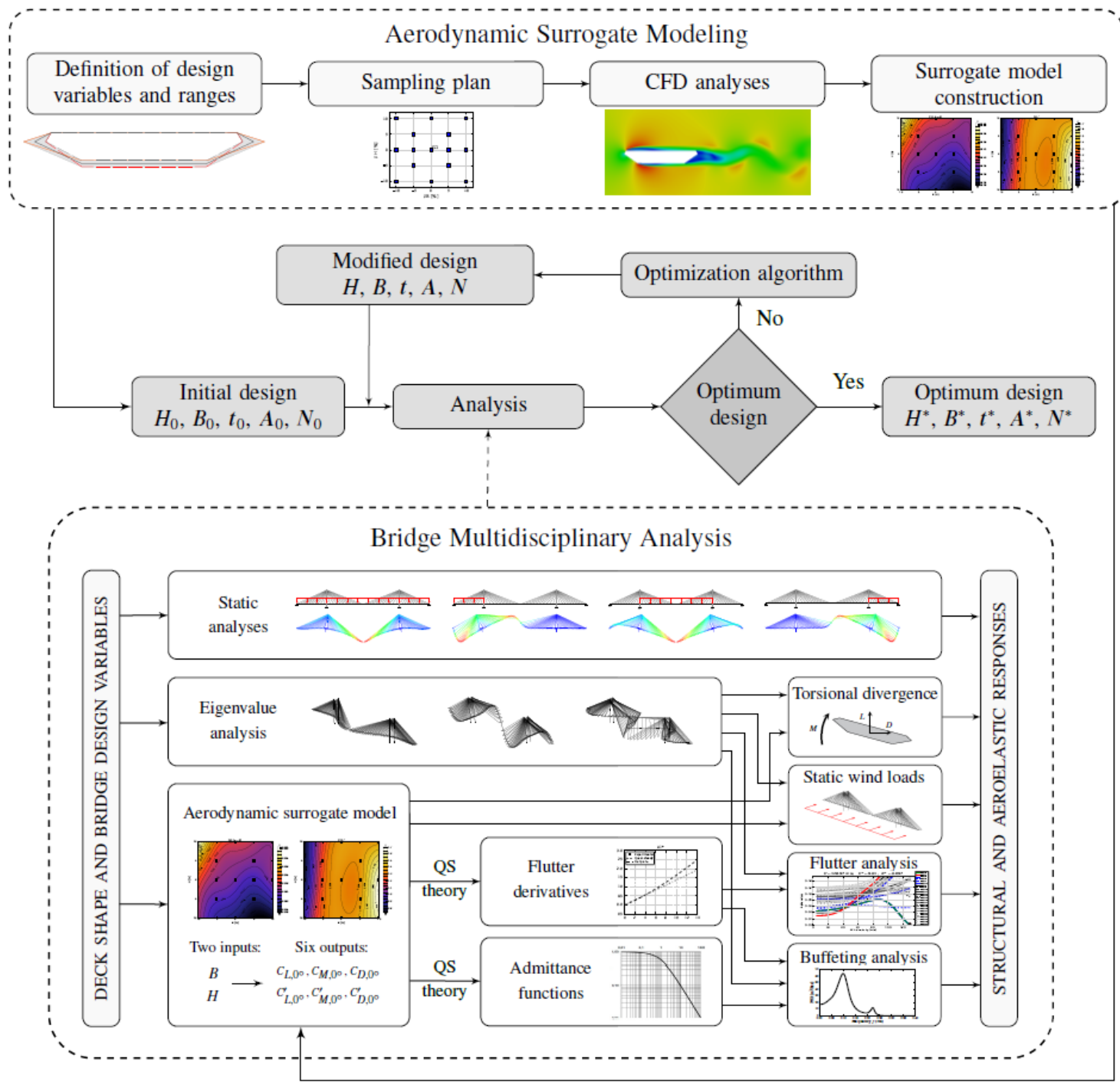

Figure 11: Flowchart of the aerostructural optimization of long span bridges

On the contrary, the joint consideration of all sets of conditions is much convenient and, of course, the optimization process guarantees that the best solution for the number of constraints of the problem is obtained and the amount of material required for the sizing of the bridge is minimized. Consequently, it can be asserted that the optimization approach is a very rational tool for engineering design (Vanderplaats 2007, Arora 2017), so the application of this methodology to any kind of structures and especially to the difficult and expensive ones (Hernandez and 
Fontan 2002) improves the design skills of the practitioners and accelerates their learning curve.

The aim of minimize the amount of material needed for the constructions of the bridge not only has financial advantages, this is also an example of sustainable design. In fact, Lucien Schmit, the researcher that pioneered, and almost created this discipline stated ".. After all, minimum weight structural design could be viewed as an allocation of scarce resources.. "(Schmit 1981). When these words were written, quite probably, the term sustainability did not exist, or at least almost nobody was aware of it.

Initial research in the application of optimization methods to aeroelastic design of bridge started some years ago (Jurado et al. 2011), and the aerostructural shape optimization problem considering the flutter velocity as design constraint has been recently addressed (Cid Montoya et al. 2018a, 2018b). A flowchart describing graphically the current state of the methodology is presented in Figure 11 (Cid Montoya et al. 2019a). It shows the different techniques required, the nature of the loads included, the classes of structural analysis carried out, some of the aeroelastic phenomena than can be considered, the design variables defined in the formulation and the description of the iterative procedure inherent to optimization algorithms.

\section{ENHANCEMENTS AND CHALLENGES FOR THE AEROSTRUCTURAL OPTIMIZATION APPROACH}

The previous sections have described the methodology of aerostructural optimization of long span bridges and the current capabilities. Nevertheless, some issues need to be incorporated to allow its application to a long span bridge that can be designed in the near future. Some can be defined as enhancements and could be added with no special difficulty, while other need an important research effort and can be termed of challenges. Thus, the last part of this paper is devoted to relate both types of future additions to the current formulation.

\section{ENHANCEMENTS TO THE CURRENT FORMULATION OF AEROSTRUCTURAL OPTIMIZATION OF LONG SPAN BRIDGES}

As mentioned before, the current formulation includes the optimization of the deck shape and the cable system, thus, additional components of the construction could be incorporated. Furthermore, some circumstances that worsen the strength of the structure could also be considered. Some examples of both concepts are next presented and can be included in future formulations.

Inclusion of towers in the formulation: So far, towers are included, obviously, in the structural model of the bridge and the horizontal displacements at the top are included in the constraints, but no design variables are defined in this part of the bridge, nor the weight of the tower is included in the objective problem and the stresses in the tower material are not included in the constraints either. Therefore, a more complete formulation of the aerostructural optimization should include these considerations.

An additional issue to be mentioned is the possible existence of aeroelastic phenomena affecting to the towers. Long span bridges require very tall towers and during the design of ultralong span bridges, as the Messina Bridge with a main span of $3300 \mathrm{~m}$, it was observed that across wind vibrations could occur in the towers and because of that, specific devices aimed to eliminate such undesirable behavior should be incorporated in the lateral sides of the towers.

Damage tolerant design of bridges: Long span cable supported bridges have a structural scheme fully depending of the cable system. Therefore, any circumstance that leads to the degradation or the weakening of that system put in danger the existence of the bridge. In that 
regard, in addition to the set of studies to be carried out with the complete structural model of the bridge, some additional studies eliminating some stays in cable stayed bridges or some hangers in suspension bridges will add safety to the formulation. Such formulation is usually entitled damage tolerant design and it is already applied to other structural typologies (Cid Bengoa et al. 2018). These situations could appear for different causes, including fires, collision of vehicles with the cables or degradation of the steel for lack of maintenance.

Some accidents in bridges, not specifically of long span length, as the case of the collapse of the cable stayed bridge in Genoa in 2018, or the recent collapse of a bowstring arch bridge in China, both due to the failure of part of the cable system are examples of this situations. Even the collapse of the Tacoma Narrows contemplated such circumstance. That day the bridge vibrated more than one hour under a mild windstorm and at some moment the excessive displacements produced by flutter led to the failure of some hangers and that event weakened the structural scheme and accelerated the collapse of the bridge.

Probabilistic approaches: Aeroelastic design of long span bridges in prone to many sources of uncertainties: Wind characteristics at bridge location, aeroelastic properties of deck or the amount of the structural damping of the full bridge are not by nature deterministic. Recently, some work has been done (Kusano et al 2018, Cid Montoya et al. 2019b) in that matter. But, in addition to that, approaches taking into account the randomness of these parameters and aimed to perform a complete reliability based formulation of the aerostructural optimization of the deck shape, cable system and bridge towers need to be included in the future.

\section{CHALLENGES TO AEROSTRUCTURAL OPTIMIZATION OF LONG SPAN BRIDGES}

Two main considerations need to be sorted out in the road of having a formulation that can represent adequately the context of the aerostructural design of a long span bridge and to achieve it with only numerical techniques, avoiding any kind of experimental techniques. They are related to the accuracy of CFD simulations and the similarity of the geometry of the deck considered in the formulation and the shape of the real deck of the bridge.

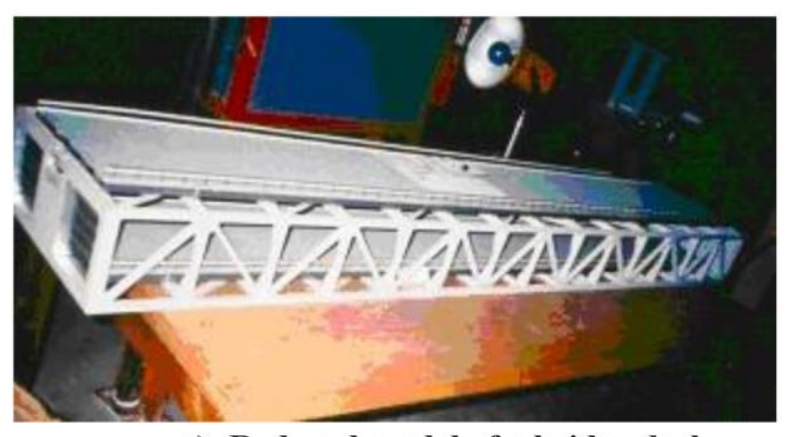

a) Reduced model of a bridge deck

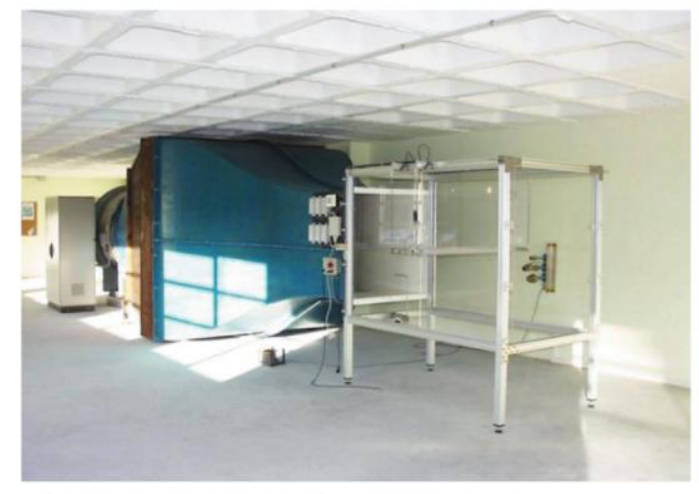

b) Test chamber of wind tunnel

Figure 12. Reduced model and aerodynamic wind tunnel (U. of Coruna)

Accuracy of CFD simulations: Although the bridge deck composed by one or two closed boxes is denominated aerodynamic deck these geometries are much less aerodynamics that the geometries used in aeronautical or car engineering. An aircraft wing is designed with a very smooth shape and the same can be said for the external configurations of cars. On the contrary a 
bridge deck, even in its simplified geometry as shown in Figure 8, has several sharp corners and the ratio depth/length is higher than in an aircraft wing. That means a bridge deck is more a bluff body than an aerodynamic one and when is immersed in a moving fluid generates turbulent flow with several scales of vortexes. This circumstance compromises the efficiency of CFD simulations and the accuracy of the results. Because of that, there is an intense activity in several research groups trying to generate more efficient turbulence formulations and modeling techniques that eliminate the current inconveniences.

Similarity between deck model and real deck geometry: One of the advantages of the tests of reduced segments of bridge deck in wind tunnels is the geometric scale allows the reproduce most details of the real bridge, safety barriers or guide vanes for instance, so the results of the experiment are extremely useful for the designers. Figure 12.a shows a reduced model used in a test in the wind tunnel of Figure 12.b.

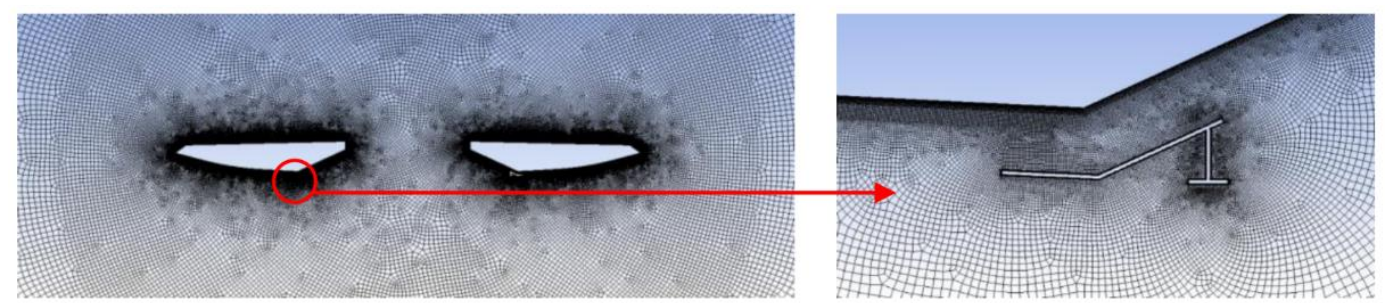

a) Bridge deck model

b) Detail of the maintenance rail

Figure 13. Detailed models of bridge deck in CFD simulations

In the case of CFD simulations is clear that the external shape of the deck can be modeled with as much detailed as wished and Figure 13.a shows an example of a deck with details that include even the maintenance rail placed under the bottom plate. But nevertheless, only bare geometries are used usually in the simulations due to the increase of the refinement required for the discretization of the domain and the difficulty to evaluate the perturbations produced in the wind flow. Thus, this is also an exciting field that requires a strong research effort.

\section{CONCLUSION}

Many cable supported bridges of long span have been built in different locations in the past decades and this trend will continue in the future with even more challenging designs. Such constructions have great social impact and are very expensive. Hence, they need to be designed with the most up to date technologies.

The conventional procedure of conceptual design of these typologies is based in an iterative process combining computational analysis and experimental studies in wind tunnels, but this approach is very cumbersome and not many prototypes can be taken into consideration. Most of these inconveniences can be eliminated by substituting that approach for another one based in fully computational calculations using CFD simulations, design of experiments and surrogate models. These technologies can constitute a framework that linked with optimization algorithms provides the best design of the bridge that accomplishes the set of structural an aeroelastic constraints included in the formulation. A description of the current capabilities of this methodology has been made and some possible improvements and weaknesses that need to be addressed in the future have been mentioned. 


\section{ACKNOWLEDGEMENTS}

This research has been funded by the Spanish Ministry of Economy and Competitiveness in the frame of the research project BIA2016-76656-R and the Galician regional government (including FEDER Funding) reference ED431C 2017/72. M. Cid Montoya has been funded by the Galician regional government (Xunta de Galicia) with reference ED481B 2018/053 and the Fulbright postdoctoral scholarship program

\section{REFERENCES}

Álvarez, A. J., Nieto, F., Kwok, K.C.S, Hernández, S. (2018) “Aerodynamic performance of twin-box decks: A parametric study on gap width effects based on validated 2D URANS simulations", Journal of Wind Engineering and Industrial Aerodynamics, Vol. 182, 202-221. Arora, J. (2017). Introduction to Optimum Design. Academic Press. Fourth Edition.

Brown, W. C. (1975a). "Long span suspensión bridges: A british approach", Annals of the New York Academie of Sciences, 352(1):1

Brown, W. C., Parsons, M. F. and Knox, H. S. G. (1975b). "Bosphorus bridge. Part 1: History of design. Part 2: Construction of superstructure". Proceedings of the Institution of Civil Engineers, ASCE, Reston, VA, volume 58, 505-567.

Bruno, L. Salvetti, M.V., Ricciardelli, F. (2014), "Benchmark on the Aerodynamics of a Rectangular 5:1 Cylinder: An overview after the first four years of activity", Journal of Wind Engineering and Industrial Aerodynamics, Vol. 126, 87-106.

Cid Bengoa, C., Baldomir, A., Hernandez, s., Romera, L. (2018). "Multi-model reliability-based design optimization of structures considering the intact configuration and several partial collapses", Structural and Multidisciplinary Optimization, Vol. 57, 977-974.

Cid Montoya, M., Nieto, F., Hernández, S., Kusano, I., Álvarez, A. J. and Jurado, J. Á. (2018a) "CFD-based aeroelastic characterization of streamlined bridge deck cross-sections subject to shape modifications using surrogate models." Journal of Wind Engineering and Industrial Aerodynamics, 177:405-428.

Cid Montoya, M., Hernández, S., and Nieto, F. (2018b) "Shape optimization of streamlined decks of cable-stayed bridges considering aeroelastic and structural constraints." Journal of Wind Engineering and Industrial Aerodynamics, 177:429-455.

Cid Montoya, M., Hernandez, S., Nieto, F., Kareem, A., Jurado J.A. (2019a), “Aerostructural Shape and Size Optimization of a Long-span Cable-stayed Bridge Considering Service Loads, Static Wind Loads, Torsional Divergence, Flutter and Buffeting Constraints." Proceedings of the 15th International Conference in Wind Engineering, Beijing, China.

Cid Montoya, M., Ding, F., Kareem, A., Hernández, S., Nieto, F., Baldomir, A. (2019b). “A reliability based tailoring of bridge deck shape for buffeting response." The 15th International Conference on Wind Engineering, Beijing, China.

Forrester, A., Sobester, A. and Keane, A. (2008), Engineering design via surrogate modelling. John Wiley and Sons, Ltd.

Hernandez, S. and Fontan, A. N., (2002). Practical Applications of Design Optimization, WIT Press.

Jurado J. A., Hernández S., Nieto N. and Mosquera A. (2011) Bridge Aeroelasticity. Sensitivity Analysis and Optimal Design, WIT Press, Ashurst Lodge, Ashurst, Southampton, SO40 7AA, UK.

Kusano, I. Baldomir, A., Jurado, J. A. and Hernandez, S. (2018), "The importance of correlation 
among flutter derivatives for the reliability based optimum design of suspension bridges". Engineering Structures, Vol. 173, 416-428

Nieto, F., Owen, J.S., Hargreaves, D.M., Hernández, S., (2015), "Bridge deck flutter derivatives: Efficient numerical evaluation exploiting their interdependence", Journal of Wind Engineering and Industrial Aerodynamics, Vol. 136, 138-150.

Nieto, F., Casteleiro A., Cid Montoya, M., Hernandez, S., Kusano, I., Jurado J.A., Fontan, A., Alvarez, A. J., Rapela, C. (2019) "Surrogate-based characterization of the aerodynamic response of twin-box decks considering changes in the box geometry and gap distance." Proceedings of the 15th International Conference in Wind Engineering, Beijing, China.

Roberts, G (1968). "Severn bridge: Design and contract arrangements. (Includes appendices)." Proceedings of the Institution of Civil Engineers, ASCE, Reston, VA, volume 41, 1-48.

Scanlan, R.H and Tomko, J.J (1971). "Airfoil and Bridge Deck Flutter Derivatives", Journal of the Engineering Mechanics Division, ASCE, Reston, VA, Vol. 97, Issue 6, 1717-1737.

Scott, R. (2001. In the Wake of Tacoma: Suspension Bridges and the Quest for Aerodynamic Stability ASCE Press, ASCE, Reston, VA.

Vanderplaats, G. N. (2007), Multidiscipline Design Optimization. VR\&D, USA.

Vickery, B.J. and Basu, R. (1983). "Across-Wind Vibrations of Structures of Circular CrossSection. Part I. Development of a mathematical model for two-dimensional conditions", Journal of Wind Engineering, 49-73. 